\title{
Genesis of the T wave as based on an Equivalent Surface Source Model.
}

\author{
A. van Oosterom \\ Department of Medical Physics, University of Nijmegen, The Netherlands \\ Published: J.Electrocardiol. Vol 34S; 2001; pp.217-227 Version: 2002-12-01; erroneous Transpose \\ corrected (twice: in Eqn. 14 and in the preceeding one) in left column page 4; \\ Appendix added, proving the equation preceding Eqn. 13
}

\begin{abstract}
This article shows the use of the equivalent surface source model in its application to the genesis of the surface ECG in which the sources are of the double layer type. This model has previously been shown to yield an accurate description of body surface potentials during the QRS interval. Its application to the genesis of the $T$ wave is now worked out in greater detail.

In this source model, the full spatio-temporal character of the cardiac electric generator is expressed by means of an equivalent double layer situated at the boundary of ventricular tissue. The timing of local depolarization and of repolarization of the cells near this boundary is used to simulate the surface ECG. The background of this source model is discussed, as well as its validity. A matrix formulation is presented of the forward problem of computing the resulting body surface potentials.

Based on this forward formulation, an inverse computation of the timing of the repolarization process was performed. Simulated $T$ waves, based on this timing, showed a close correspondence with measured body surface potentials.

By taking the inversely computed timing of repolarization as representing the true situation, the model can be used to study in which way perturbations in the timing of local repolarization effect the shape of $T$ waves. An analysis based on this model indicates that the amplitude of the $T$ wave is proportional to the dispersion of the repolarization times at the ventricular surface. The model is also shown to be capable of simulating the ECG changes during local ischemia.
\end{abstract}

Key words: $\mathbf{T}$ wave model, repolarization, dispersion.

\section{INTRODUCTION}

The correct interpretation of $\mathrm{T}$ wave abnormalities requires a model of the genesis of the normal $\mathrm{T}$ wave against which observed wave forms can be contrasted. The ECG wave forms as observed at some distance from the active tissues can only be interpreted correctly in a physiological sense if appropriate models are incorporated of the cardiac electric sources and of the volume conduction effects of the surrounding tissues.

Cardiac sources have a spatio-temporal character. The temporal features dominate the potentials observed at a distance and tend to obscure the spatial features. This holds true in particular during the repolarization process. Most ECG textbooks include some notions related to the genesis of the $\mathrm{T}$ wave. Correct as these may be in a general sense, they do not do sufficient justice to the full complexity of the problem. More comprehensive discussions on this topic can be found in [1], [2].

In this study the full spatio-temporal character of the cardiac electric generator is expressed by means of an equivalent double layer situated at the boundary of ventricular tissue. The timing of local depolarization and of repolarization of the cells near this boundary is used to simulate the surface ECG. This approach is

\footnotetext{
${ }^{1}$ Address for correspondence: A. van Oosterom, Department of Medical Physics, Geert Grooteplein 21, 6525EZ, Nijmegen, The Netherlands. E-mail: avo@mbfys.kun.nl
}

essentially similar to one shown in the pioneering study on this topic by Harumi et al. [3]. The formal justification of the source model was provided by Geselowitz [4], [5].

When combined with a realistic volume conductor model of the thorax, the equivalent surface model has initially been shown to yield an accurate description of the potentials during QRS [6], [7]. At a later stage the source model was applied to the modeling of the $\mathrm{T}$ wave [8]. The present paper explores this approach in some greater detail.

First, the source model used is briefly summarized and some comments are made on its validity. Next, a matrix formulation is presented of the forward problem of computing the resulting body surface potentials. It is shown how this formulation can be incorporated in an inverse procedure aimed at recovering the timing of depolarization as well as that of repolarization. An inversely computed sequence of repolarization is presented. Finally, some model based interpretations of $\mathrm{T}$ wave properties are presented.

\section{THEORY}

\section{A. The Equivalent Surface Source of type UDL}

In this equivalent surface source model the entire electrical activity within the ventricles is expressed by means of a double layer on the heart surface $S_{\mathrm{h}}$, the surface bounding all ventricular tissue. For any position on $S_{\mathrm{h}}$ the local source strength is proportional to the transmembrane potential $\varphi_{m}$ of the cells near the boundary. With the maximum strength at any point on $S$ assumed to be the same, it leads to a uniform double layer (UDD). In his paper of 1989 Geselowitz [4] derived this result from an analysis of the electrical properties of ventricular cells treated as a homogeneous syncytium by means of the bidomain approach. In that paper the key result (his Eqn. 63) of the bidomain approach was shown to be

$\varphi(\vec{y})=-\int_{v o l} g_{i} \nabla \varphi_{m}(\vec{x}) \cdot \nabla Z d v$

with $\varphi(\vec{y})$ the potential in some observation point $\vec{y}$ outside the heart, $g_{i}$ the conductivity of the intracellular aspect of the bidomain, $\nabla$ the gradient operator, $\varphi_{m}(\vec{x})$ the transmembrane potential at all points $\vec{x}$ throughout the heart and $Z=Z(\vec{y}, \vec{x})$ the lead field of the electrode configuration involved. This equation expresses the summation of the contributions to the external potential of all local transmembrane potential differences (gradients) 
present throughout the entire volume, vol, of the ventricular tissue.

Next, by applying vector calculus to Eqn. 1 and by using the properties of $Z$, Geselowitz showed that

$\varphi(\vec{y})=-\int_{S_{\mathrm{h}}} g_{i} \varphi_{m}(\vec{x}) \nabla Z \cdot d \overrightarrow{S_{\mathrm{h}}}$,

(his Eqn. 68), now involving an integration over the surface $S_{\mathrm{h}}$ and $\vec{x}$ specifying the source location on $S_{\mathrm{h}}$. For potentials $\varphi_{\infty}$ in an hypothetical, infinite homogenous medium surrounding the heart, $Z$ can be shown to be

$Z(\vec{y}, \vec{x})=\frac{1}{4 \pi \sigma_{o} R}$

with $\sigma_{0}$ the conductivity of the medium and $R$ the distance between $\vec{x}$ and $\vec{y}$ (see, e.g., [9]). Substitution of Eqn. 3 in Eqn. 2 leads to

$\varphi_{\infty}(\vec{y}, t)=-\frac{g_{i}}{4 \pi \sigma_{0}} \int_{S_{\mathrm{h}}} \varphi_{m}(\vec{x}, t) \nabla \frac{1}{R} \cdot d \overrightarrow{S_{\mathrm{h}}}$.

This expression is valid at all time instants $t$. In deriving this result Geselowitz used the boundary condition $g_{i} \nabla \varphi_{m} \cdot d \overrightarrow{S_{\mathrm{h}}}=0$, a boundary condition also used in the work of Colli-Franzone et al.[10] (see also the discussion on this boundary condition in Chapter 5.9 of [9]).

The term $\nabla \frac{1}{R} \cdot d \overrightarrow{S_{\mathrm{h}}}$ in Eqn. 4 is the solid angle $d \omega$ subtended by the elementary surface element $d S_{\mathrm{h}}(\vec{x})$ at $\vec{y}$, and so we find

$\varphi_{\infty}(\vec{y}, t) \propto-\int_{S_{\mathrm{h}}} \varphi_{m}(\vec{x}, t) d \omega(\vec{y}, \vec{x})$.

In his 1989 paper Geselowitz stipulated that the bidomain should be isotropic, but in his 1992 paper he showed that this restriction is lifted for situations where the ratio of extracellular to intracellular conductivity in the fiber direction can be taken to be the same as that in the cross-fiber direction. This is the assumption that dominates the application of the equivalent surface source model. The justification for this approach stems from the fact that the measured wave front strength during depolarization in the extracellular part of the ventricles in the fiber direction is approximately the same as that in the cross-fiber direction (40 mV; see [11], [12], [13]).

Regarding the depolarization phase we previously arrived at the equivalence of the sources at the actual wavefront of depolarization and hypothetical sources situated on $S_{\mathrm{h}}$. At that stage [14] the equivalence was derived from the classical solid angle theory ([15], [12]). Equation 5 legitimizes the use of the equivalent surface source applied to repolarization in situations for which the implied assumptions hold true. Simulated T waves based on Eqn. 5 have previously been reported in the literature ([8], [16], [17]).

\section{B. Matrix formulation of the Forward Problem}

The human body is clearly not infinite: it is a bounded inhomogeneous, anisotropic volume conductor. Its effects on potentials at some distance from the source can be expressed as a linear operator acting on the infinite medium potentials (see, e.g., [9]). As a consequence the potential at any point $\vec{y}$ within the thorax or on the body surface reads

$\varphi(\vec{y}, t) \propto-\int_{S_{\mathrm{h}}} A(\vec{y}, \vec{x}) \varphi_{m}(\vec{x}, t) d \omega(\vec{y}, \vec{x})$

with $A(\vec{y}, \vec{x})$ expressing the full complexity of the volume conductor (geometry and conductivity).

Equation 6 uses the continuous variables of position $\vec{y}$ and $\vec{x}$. The complexity of the geometry does not allow one to work out Eqn. 6 by means of an analytical method. Instead, in a numerical approach, discretized versions of $\vec{x}$ and $\vec{y}$ were used: a collection of nodes $x_{n} ; n=1, N$ representing the source positions and a collection of points $y_{\ell} ; \quad \ell=1, L$ representing the field points (leads). Similarly, time was discretized in $T$ time instants $t$.

We now introduce a matrix $\mathbf{A}$, having elements $a_{\ell, n}=-A\left(y_{\ell}, x_{n}\right) d \omega\left(y_{\ell}, x_{n}\right)$. In addition we introduce a matrix $\mathbf{S}$ having elements $s_{n, t}=\varphi_{m}\left(x_{n}, t\right)$ and a matrix $\boldsymbol{\Phi}$, of dimension $L \times T$, the columns of which represent the instantaneous (body surface) potentials at all $T$ subsequent time instances, whereas the rows represent the time tracings of the $L$ field points. Note that, similarly, the rows of $\mathbf{S}$ represent the temporal aspects of the source strength around the $N$ nodes on $S_{\mathrm{h}}$; its columns represent the spatial distribution of the source strength over $S_{\mathrm{h}}$ at subsequent time instants.

The use of matrixes $\mathbf{A}$ and $\mathbf{S}$ makes that Eqn. 6 can be approximated by

$\boldsymbol{\Phi}=\mathbf{A} \mathbf{S}$

Throughout this paper matrixes as well as vectors in the sense of linear algebra are printed in bold face. The dependence of a matrix $\mathbf{S}$ on the elements of a vector $\boldsymbol{\rho}$ is denoted as $\mathbf{S}\{\boldsymbol{\rho}\}$.

The expression by Eqn. 7 can be used for any type of distributed source. The linearity of the multiplication by matrix A stems from the linearity of the conductive properties of the medium. In vectorcardiography just $N=3$ sources are involved (the three orthogonal components of the dipole). In that application the rows of $\mathbf{A}$ are called the (3-D) lead vectors. For larger values of $N$, as is the case in this paper, the $N$-dimensional row vector $A_{\ell, \text {. }}$ expresses the linear weighting of all instantaneous source strengths for lead $\ell ; \ell=1, L$.

For the equivalent surface sources as used here $\mathbf{A}$ has the following important property: the sum of all elements of any row is zero. This stems from the fact that the solid angle subtended by a closed surface at any exterior point is zero. This property can be formulated as

$\mathbf{A} \mathbf{e}=\mathbf{o}$ 
with e an $N$-dimensional column vector having unit elements only, and o an $L$-dimensional column vector having zero elements only. Expressed in matrix terminology: A has a zero eigenvalue with a corresponding right eigenvector $\mathbf{e}$.

This property implies that at any point in time the mean value of the source strength over $S_{\mathrm{h}}$ does not generate any potential difference outside $S_{\mathrm{h}}$.

\section{Materials And Methods}

The matrix formulation Eqn. 7 allows one to simulate body surface potentials throughout the QRST interval. For its use one needs to specify source matrix $\mathbf{S}$ as well as transfer matrix $\mathbf{A}$. In this paper all data relate to a single subject. The computation from observed body surface potentials of the depolarization se-

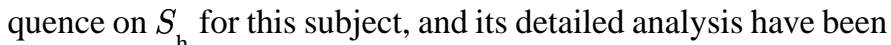
documented before ([6], [7]). This paper extends the analysis to the time interval of repolarization.

\section{A. Measured potentials}

The body surface potentials of the subject were recorded using the $L=64$ leads of the Nijmegen lead system [18]. This lead system has the positions of the standard 12-lead ECG as a subset. A $500 \mathrm{~ms}$ interval of QRST signals was sampled at a rate of 500 samples/s. The data were stored in a matrix $\mathbf{V}$ of dimension $64 \times 250$. In our previous work the measured data were restricted to the QRS interval, which were assumed to be unaffected by early repolarization. Similarly, we now assume the remaining part of the measured ECG data as being generated exclusively by the repolarization currents. Because of this we can partition $\mathbf{V}$ as $\mathbf{V}=\left[\mathbf{V}_{\mathrm{d}} \mathbf{V}_{\mathrm{r}}\right]$, with the subscripts $\mathrm{d}$ and $\mathrm{r}$ denoting the depolarization phase (QRS interval; $100 \mathrm{~ms}$ ) and the repolarization phase (ST interval; $400 \mathrm{~ms}$ ), respectively. Accordingly, matrix $\mathbf{V}_{d}$ was of dimension $64 \times 50$ and $\mathbf{V}_{\mathbf{r}}$ was of dimension $64 \times 200$. Separate linear baseline corrections were performed on both time intervals, to make the data consistent with the assumed absence of simultaneous depolarization and repolarization.

\section{B. Specifying A}

Matrix A was the same as the one used in our previous work on the depolarization phase. It represents the transfer from the elementary double layer sources around $N=257$ nodes evenly distributed over the surface $S$ to $L=64$ leads on the thorax. The individual columns of $\mathbf{A}$ were referenced to zero mean.

A piecewise homogeneous, multi-compartmental volume conductor model was involved in the computation of $\mathbf{A}$, specifying the inhomogeneity interfaces of the lungs and the bloodfilled cavities. The conductivity values of these compartments relative to the overall conductivity of the body, were: lungs 0.2 , cavities 3.0. The geometry of these compartments was extracted from MRI data of the (healthy, male) subject. The transfer was computed by means of the Boundary Element Method. For more details, see [6], [7], [19]. The matrix A was of dimension $64 \times 257$.

\section{Specifying $\mathbf{S}$}

In this work the time course of the source strength at any of the nodes on $S_{\mathrm{h}}$ has been assigned the shape depicted in Fig. 1.

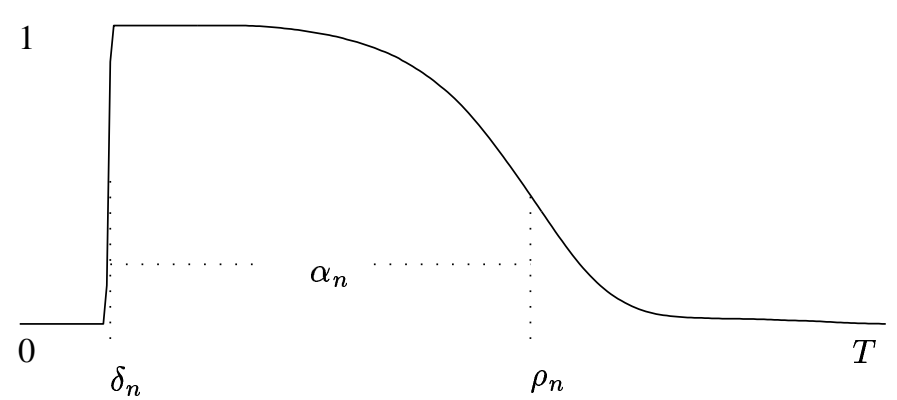

Fig. 1. Time course of the source strength $S_{n}$ at node $n$.

This curve approximates the typical time course of the transmembrane potential of a ventricular muscle cell. Details of its actual shape are presented in Sect. III-E. The general shape of this curve is assigned to all nodes. The time instant of the maximum positive slope is taken as specifying the moment of local depolarization. For node $n$ it is denoted as $\delta_{n}$, element $n$ of a vector $\boldsymbol{\delta}$. The time instant of maximum negative slope is taken as a marker for the timing of local repolarization. For node $n$ it is denoted as $\rho_{n}$, element $n$ of a vector $\boldsymbol{\rho}$. The interval $\alpha_{n}=\rho_{n}-\delta_{n}$ is a measure of the local duration of the transmembrane potential, the action potential duration (APD) [20]. We take $\alpha_{n}$ to be an element of a vector $\boldsymbol{\alpha}$.

The double layer strength used $(40 \mathrm{mV})$ is assumed to be the same for all nodes. Its value stems from the amplitude of the upstroke of the transmembrane potential, scaled by $g_{i} / \sigma_{0}$ (Eqn. 4 ). This value is incorporated in the scaling of $\mathbf{A}$, and so a normalized (dimensionless) maximum value of 1 can be used for the local source strength around the nodes, the value shown in Fig. 1.

As a consequence of Eqn. 8 the resting value of the source strength can be taken as zero.

Following this source specification $\mathbf{S}$ is denoted as $\mathbf{S}\{\boldsymbol{\delta}, \boldsymbol{\rho}\}$, with all its elements $s_{n, t}$ depending on $\boldsymbol{\delta}$ and $\boldsymbol{\rho}$ only, and the entire formalism for simulating body surface potentials from the surface source model reads

$\boldsymbol{\Phi}=\mathbf{A} \mathbf{S}\{\boldsymbol{\delta}, \boldsymbol{\rho}\}$

\section{Specifying the timing}

To complete the model we need to specify $\boldsymbol{\delta}$ and $\boldsymbol{\rho}$. Both vectors were obtained by means of an inverse computation, in which simulated potentials $\boldsymbol{\Phi}=\mathbf{A} \mathbf{S}\{\boldsymbol{\delta}, \boldsymbol{\rho}\}$ were matched in the least squares sense to the measured potentials $\mathbf{V}$. The computation of the timing of depolarization, $\boldsymbol{\delta}$, was presented in our earlier work. For the timing of repolarization a preliminary result was included in [8]. The inverse method used has now been adapted to take into account the special nature of the repolarization process. A brief description of the method is as follows.

The source strength $\mathbf{S}$ depends non-linearly on $\boldsymbol{\delta}$ and $\boldsymbol{\rho}$, as a consequence so does $\boldsymbol{\Phi}$. Correspondingly, the least squares problem of computing $\boldsymbol{\delta}$ and $\boldsymbol{\rho}$ such that the difference between the measured $\mathbf{V}$ and the simulated $\boldsymbol{\Phi}$ is minimal, is a non-linear 
parameter estimation problem. As a measure of this difference we take the sum of the squared differences of all elements of $\mathbf{V}$ and $\boldsymbol{\Phi}: E=\|\mathbf{V}-\boldsymbol{\Phi}\|_{\mathrm{F}}^{2}$, the squared Frobenius norm of $\mathbf{V}-\boldsymbol{\Phi}$.

The same as was done for the matrix of measured potential, the matrix $\mathbf{S}$ is partitioned as $\mathbf{S}=\left[\mathbf{S}_{\mathrm{d}} \mathbf{S}_{\mathrm{r}}\right]$. This means that the ba-

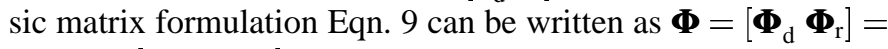
$\mathbf{A S}=\mathbf{A}\left[\begin{array}{ll}\mathbf{S}_{\mathrm{d}} & \mathbf{S}_{\mathrm{r}}\end{array}\right]=\left[\begin{array}{lll}A \mathbf{S}_{\mathrm{d}} & \mathbf{A} \mathbf{S}_{\mathrm{r}}\end{array}\right]$. In our previous work the measured data were restricted to the QRS interval and we solved $\boldsymbol{\delta}$ from arg min $\left\|\mathbf{V}_{\mathrm{d}}-\mathbf{A} \mathbf{S}_{\mathrm{d}}\{\boldsymbol{\delta}\}\right\|_{\mathrm{F}}^{2}$. In a similar way we now solve $\boldsymbol{\rho}$ from $\arg \min \left\|\mathbf{V}_{\mathrm{r}}-\mathbf{A} \mathbf{S}_{\mathrm{r}}\{\boldsymbol{\rho}\}\right\|_{\mathrm{F}}^{2}$, the least squares solution to

$\mathbf{V}_{\mathrm{r}}=\mathbf{A} \mathbf{S}_{\mathrm{r}}\{\boldsymbol{\rho}\}$.

The solution method used in this paper is the well-known Levenberg-Marquardt algorithm [21]. The first steps of this method are described here in some detail, denoted for solving $\boldsymbol{\rho}$, since it provides an interesting view on the genesis of the $\mathrm{T}$ wave. To simplify the notation we drop the subscript $r$ when dealing with repolarization only.

We now introduce a function $\mathscr{D}(u)$ of the dimensionless variable $u=\left(T \rho_{n}\right) / w$, with $1 / w$ representing the absolute value of the maximum slope of the scaled variant of the transmembrane potential during depolarization shown in Fig. 1. In this way, $\mathscr{D}(0)$ is the (dimensionless) source strength at the moment of maximal down-slope, $\frac{d}{d u} \mathscr{D}(0)=-1$ and for the elements of matrix $\mathbf{S}$ we find

$s_{n, t}=(\mathscr{D}(u))_{u=\left(T \rho_{n}\right) / w}$.

In the Marquardt algorithm, starting from an initial guess about the solution, $\boldsymbol{\rho}_{0}$, subsequent adjustments $\Delta \boldsymbol{\rho}_{k} ; k=1, \cdots$ are computed iteratively such that, by adding it to the previous estimate, the value of $E$ is reduced at each iteration. At iteration $k$ the adjustment $\Delta \boldsymbol{\rho}_{k}$ is found as the least squares solution to

$\mathbf{V}=\mathbf{A} \mathbf{S}\left\{\boldsymbol{\rho}_{k-1}+\Delta \boldsymbol{\rho}_{k}\right\}$

To solve this problem, $\mathbf{S}$ is expressed by means of a Taylor expansion at the previous iterate $\boldsymbol{\rho}_{k-1}$. Taking just the first two terms of this expansion (the first order approximation) leads to

$$
\mathbf{S}\left\{\boldsymbol{\rho}_{k-1}+\Delta \boldsymbol{\rho}_{k}\right\} \simeq \mathbf{S}\left\{\boldsymbol{\rho}_{k-1}\right\}+\mathbf{D}\left\{\Delta \boldsymbol{\rho}_{k}\right\} \mathbf{S}^{\prime}\left\{\boldsymbol{\rho}_{k-1}\right\},
$$

with $\mathbf{D}$ denoting a diagonal matrix having the elements of $\Delta \boldsymbol{\rho}_{k}$ as its diagonal, and $\mathbf{S}^{\prime}$ a matrix comprising the gradients of $\mathbf{S}$ with respect to $\boldsymbol{\rho}$. The relative simplicity of this expression stems from the fact that differentiation of the elements of $\mathbf{S}$ with respect to any $\rho_{m}$ has a non-zero effect on the elements of row $m$ only. For the elements of $\mathbf{S}^{\prime}$ we find

$s_{n, t}^{\prime}=-\frac{1}{w}\left(\frac{d}{d u} \mathscr{D}(u)\right)_{u=\left(T \rho_{n}\right) / w}$.

Recall that $\frac{d}{d u} \mathscr{D}(0)=-1$ and, hence, $\left(s_{n, t}^{\prime}\right)_{t=\rho_{n}}=1 / w$.
We now combine Eqn. 11 and Eqn.12 and find, while restricting the notation to labels of the iteration steps,

$\mathbf{V} \simeq \mathbf{A} \mathbf{S}_{k-1}+\mathbf{A D}\left\{\Delta \boldsymbol{\rho}_{k}\right\} \mathbf{S}_{k-1}^{\prime}=\boldsymbol{\Phi}_{k-1}+\mathbf{A D}\left\{\Delta \boldsymbol{\rho}_{k}\right\} \mathbf{S}_{k-1}^{\prime}$.

The least squares solution of the vector $\Delta \boldsymbol{\rho}_{k}$ from Eqn. 13 was performed by interpreting all elements of the $L \times T$-dimensional matrix of potentials as well as those of matrix of residuals, $\mathbf{R}_{k-1}=\mathbf{V}-\boldsymbol{\Phi}_{k-1}$, as the elements of $L T$-dimensional column vectors. The vector variant of these residuals is denoted as $\mathbf{r}_{k-1}$ The general solution to this problem, an overdetermined system of linear equations, reads $\Delta \boldsymbol{\rho}_{k}=\left(\mathbf{G}_{k-1}^{T} \mathbf{G}_{k-1}\right)^{-1} \mathbf{G}_{k-1}^{T} \mathbf{r}_{k-1}$, with $\mathbf{G}_{k}$ a $L T \times N$-dimensional matrix of the gradients with respect to the $N$ elements of $\Delta \boldsymbol{\rho}_{k}$ of all $L T$ elements of $\mathbf{r}_{k-1}$, and ${ }^{T}$ and ${ }^{-1}$ denoting the transverse and the inverse of a matrix, respectively. By using the special properties of $\mathbf{S}$ as apparent from Eqn. 12, it can be shown that

$\Delta \boldsymbol{\rho}_{k}=\left(\mathbf{A}^{T} \mathbf{A} \cdot * \mathbf{S}_{k-1}^{\prime} \mathbf{S}_{k-1}^{\prime T}\right)^{-1}\left(\mathbf{S}_{k-1}^{\prime} \mathbf{R}_{k-1}^{T} \cdot * \mathbf{A}^{T}\right) \mathbf{e}$

with e the $L$-dimensional unit vector and $* *$ denoting the element by-element multiplication of matrices. Following our earlier work [6], the (spatial) smoothness of the solution $\boldsymbol{\rho}$ was guarded by constraining the norm of the Surface Laplacian of the solution over $S_{b}$. Following Marquardt, the size of each iteration step was adjusted such that $E$ indeed reduced at each iteration. By including these two constraints, the actual expression used for solving $\Delta \boldsymbol{\rho}_{k}$ is

$$
\begin{aligned}
& \Delta \boldsymbol{\rho}_{k}=\left(\mathbf{A}^{T} \mathbf{A} \cdot * \mathbf{S}_{k-1}^{\prime} \mathbf{S}_{k-1}^{\prime T}+\mu^{2} \mathbf{L}^{T} \mathbf{L}+\lambda^{2} \mathbf{I}\right)^{-1} \\
& \left(\left(\mathbf{S}_{k-1}^{\prime} \mathbf{R}_{k-1}^{T} * * \mathbf{A}^{T}\right) \mathbf{e}-\mu^{2} \mathbf{L}^{T} \mathbf{L} \boldsymbol{\rho}_{k-1}\right),
\end{aligned}
$$

in which $\mathbf{I}$ is the identity matrix of dimension $N, \mathbf{L}$ is the matrix of the discretized Surface Laplacian operator, and $\mu$ and $\lambda$ set the weights of the constraints.

The timing of the depolarization, $\boldsymbol{\delta}$, was computed in a similar manner, this time by using an activation function $\mathscr{U}(u)$ representing the up-slope of the curve shown in Fig. 1. The duration of the up-slope was put at $4 \mathrm{~ms}$, being an estimate of the mean activation time over any part of $S_{\mathrm{h}}$ represented by an individual node.

\section{E. Finding the shape of $\mathscr{D}$}

A dominant prerequisite for solving $\boldsymbol{\rho}$ is the shape of the down-slope of the transmembrane potential, $\mathscr{D}(u)$. From the theory exposed in Sect. III-D the following method was derived for estimating this shape from the measured ECG data.

We start by taking $-\boldsymbol{\rho}$ to denote a vector for which all elements have the same value $-\rho$ : the mean of the elements of $\boldsymbol{\rho}$. Any vector $\boldsymbol{\rho}$ can thus be represented as $\boldsymbol{\rho}={ }^{-} \boldsymbol{\rho}+\Delta \boldsymbol{\rho}$, with $\Delta \boldsymbol{\rho}$ representing $\boldsymbol{\rho}-\boldsymbol{\rho}$. By substitution of this expression in the forward formulation and equating the first order approximation of the Taylor expansion applied to $\mathbf{S}$, compare Eqns. 11 and 12, we now find

$\left.\mathbf{V} \simeq \mathbf{A S}\{\boldsymbol{\rho}\}=\mathbf{A S}\left\{{ }^{-} \boldsymbol{\rho}+\Delta \boldsymbol{\rho}\right\} \simeq \mathbf{A S}\left\{{ }^{-} \boldsymbol{\rho}\right\}+\mathbf{A D}\{\Delta \boldsymbol{\rho}\}^{\prime} \mathbb{S}^{-} \boldsymbol{\rho}\right\}$ 


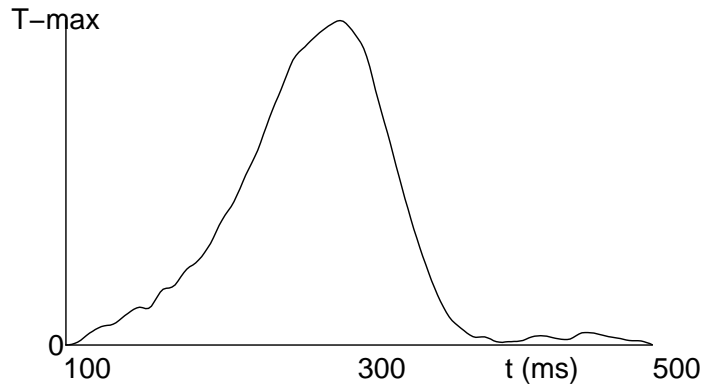

Fig. 2. Time course of $S^{\prime}(t, \bar{\rho})$ estimated from measured $\mathrm{T}$ wave data $\mathbf{V}_{\mathrm{r}}$.

The first term on the right is zero because of Eqn. 8, which means that

$\mathbf{V} \simeq \mathbf{A D}\{\Delta \boldsymbol{\rho}\} \mathbf{S}^{\prime}\left\{{ }^{-} \boldsymbol{\rho}\right\}$

All rows of $\mathbf{S}^{\prime}\left\{{ }^{-} \boldsymbol{\rho}\right\}$ are identical and we denote these as $S\left(t ;{ }^{-} \rho\right)$. For the time course of any lead $\ell$ we find

$V_{\ell}(t) \simeq S^{\prime}(t ;-\rho) \sum_{n} a_{\ell, n} \Delta \rho_{n}$

which means that, in this first order approximation, the $\mathrm{T}$ waves in all leads have the same shape, $S^{\prime}\left(t ;{ }^{-} \rho\right)$, and have amplitudes that are linear combinations of the individual $\Delta \rho_{n}$ values. This being the case we can now estimate $S^{\prime}\left(t ;{ }^{-} \rho\right)$ from the measured data $\mathbf{V}_{\mathrm{r}}$. To this end, sign changes were applied to some of the measured signals in such a way that all peak $\mathrm{T}$ wave values became positive. The sum of these adapted signals was taken as describing the shape of $S^{\prime}(t ;-\rho)$. This shape is depicted in Fig. 2. Integration of this function over time yielded, after an appropriate sign change, shifting and scaling, the function $\mathscr{D}(u)$. The result was used to specify the down-slope part of the curve shown in Fig. 1.

\section{RESUlts}

Isochrone maps of the inversely computed depolarization timing $\boldsymbol{\delta}$ and of the repolarization timing $\boldsymbol{\rho}$, drawn on various sections of $S_{\mathrm{h}}$, are shown in Fig. 3 and Fig. 4, respectively. The value of the regularization parameter $\mu$ was adjusted such that the smoothness of $\boldsymbol{\rho}$ was about the same as the smoothness previously established for $\boldsymbol{\delta}$ (see [6]). The solution $\boldsymbol{\delta}$ shown in Fig. 3 is similar to the one shown in our previous work which used the Quasi-Newton method for solving the non-linear parameter estimation problem involved. In [19] its realism is demonstrated by comparing the solution to invasively measured reference data [22]. For the solution $\boldsymbol{\rho}$ no such reference data were available. The vector of APD values at the nodes on $\mathbf{S}$ was computed as $\boldsymbol{\alpha}=\boldsymbol{\rho}-\boldsymbol{\delta}$. Some statistics of the elements of $\boldsymbol{\delta}, \boldsymbol{\rho}$ and $\boldsymbol{\alpha}$ are listed in Table 1. The line of linear regression of the elements of $\boldsymbol{\alpha}$ as a function of those of $\boldsymbol{\delta}$ demonstrated a slope of -1.43 , in agreement with the generally accepted notion that cells that depolarize late have relatively short APD values. Note that if the vectors $\boldsymbol{\delta}$ and $\boldsymbol{\rho}$ were uncorrelated the slope of $\boldsymbol{\alpha}$ vs. $\boldsymbol{\delta}$ would be -1 .
Table 1. Statistics of the elements of the solution vectors of timing: $\boldsymbol{\delta}, \boldsymbol{\rho}$ and $\boldsymbol{\alpha}$.

\begin{tabular}{cccc}
\hline $\begin{array}{c}\text { statistic } \\
(\mathrm{ms})\end{array}$ & $\begin{array}{c}\boldsymbol{\delta} \\
(\text { depol })\end{array}$ & $\begin{array}{c}\boldsymbol{\rho} \\
\text { (repol) }\end{array}$ & $\begin{array}{c}\boldsymbol{\alpha} \\
(\mathrm{APD})\end{array}$ \\
\hline mean & 48.7 & 281 & 233 \\
SD & 11.9 & 9.83 & 19.1 \\
min & 20.0 & 263 & 193 \\
max & 79.6 & 310 & 284 \\
range & 59.6 & 47 & 91
\end{tabular}
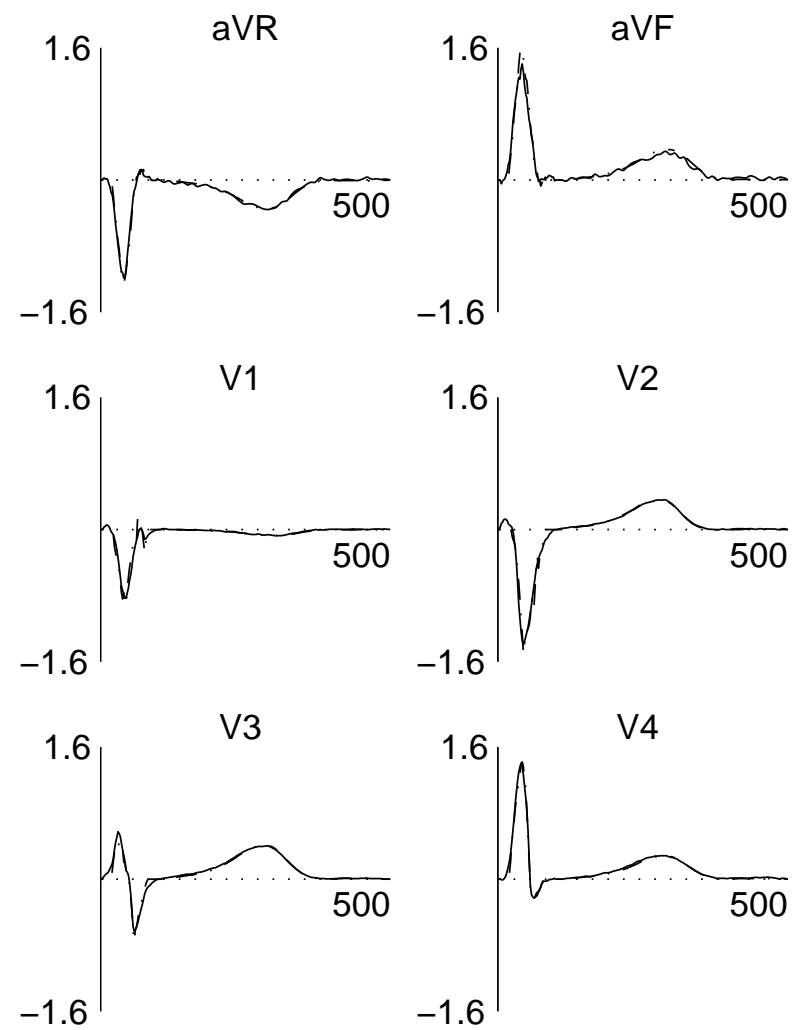

Fig. 5. Measured and simulated signals of six of the standard 12-lead ECGs; solid lines: measured signals; dash-dot: simulated signals; note that these are hardly distinguishable from the measured ones. Voltage scale: $1.5 \mathrm{mV}$; time scale: $500 \mathrm{~ms}$.

The wave forms of six of the standard 12-lead ECGs are shown in Fig. 5. The solid lines are subsets of the measured $\mathbf{V}$, the dash-dot lines depict the corresponding subsets of the simulated $\boldsymbol{\Phi}=\mathbf{A} \mathbf{S}\{\boldsymbol{\delta}, \boldsymbol{\rho}\}$. Note that the differences between these curves are small. The values of the relative residual differences, $r d=\|\mathbf{V}-\boldsymbol{\Phi}\|_{\mathrm{F}} /\|\mathbf{V}\|_{\mathrm{F}}$, computed over the QRS interval and the ST interval were 0.17 and 0.11 , respectively. The notation ST interval in this paper refers to the entire repolarization phase.

\section{AppliCATION}

The results shown in Fig. 5 demonstrate that by using the computed, geometry based transfer $\mathbf{A}$ and the inversely computed timings $\boldsymbol{\delta}$ and $\boldsymbol{\rho}$ the formalism, Eqn. 9, produces simulated $\boldsymbol{\Phi}$ data that closely correspond to the measured data $\mathbf{V}$. In this section some applications of the formalism are described. 

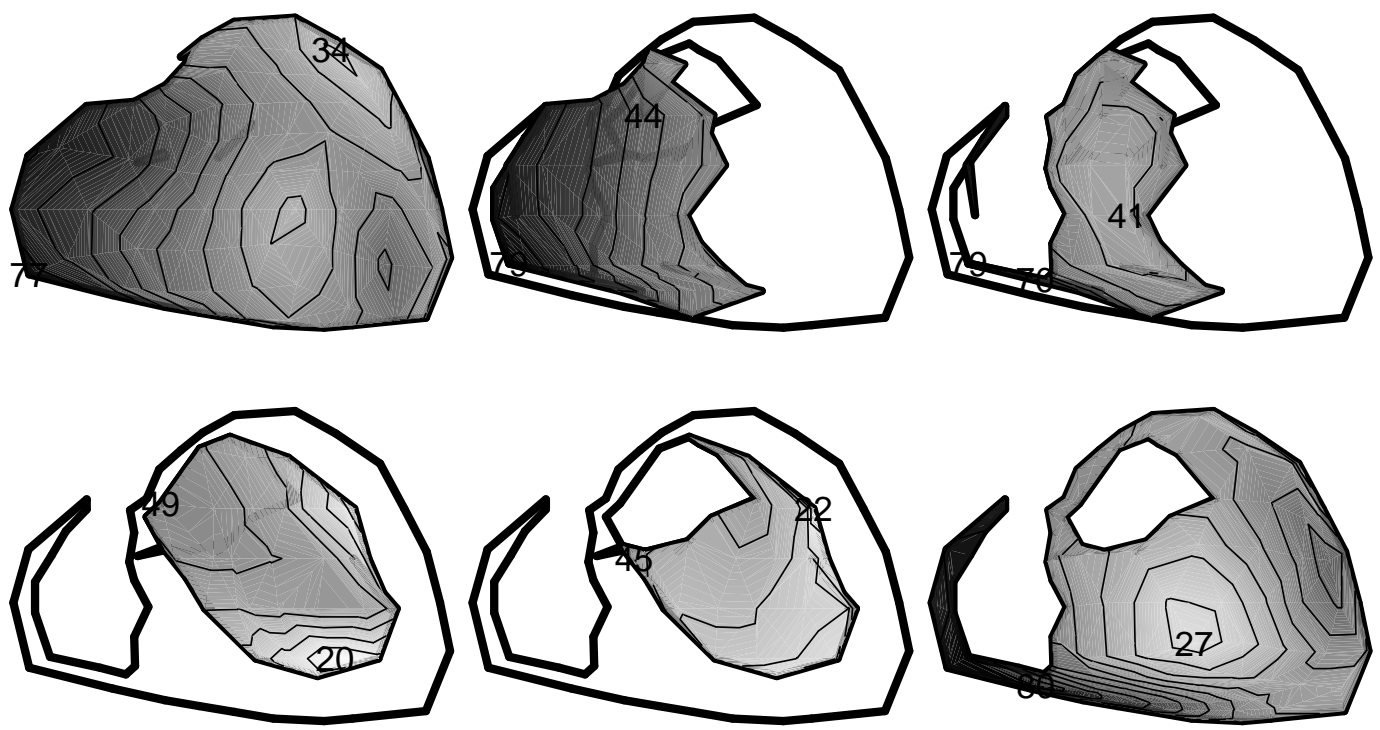

Fig. 3. Isochrone map of the inversely computed timing of depolarization, $\boldsymbol{\delta}$, plotted on different parts of $S_{\mathrm{h}}$ : Top row, left to right: anterior epicardium, endocardium right ventricle, septum right ventricle. Bottom row, left to right: septum left ventricle, endocardium left ventricle, posterior epicardium. The integers shown are milliseconds from the onset of QRS. Successive isochrones are at $5 \mathrm{~ms}$ intervals. Shading has been applied to assist in the identification of individual isochrones: darker=later
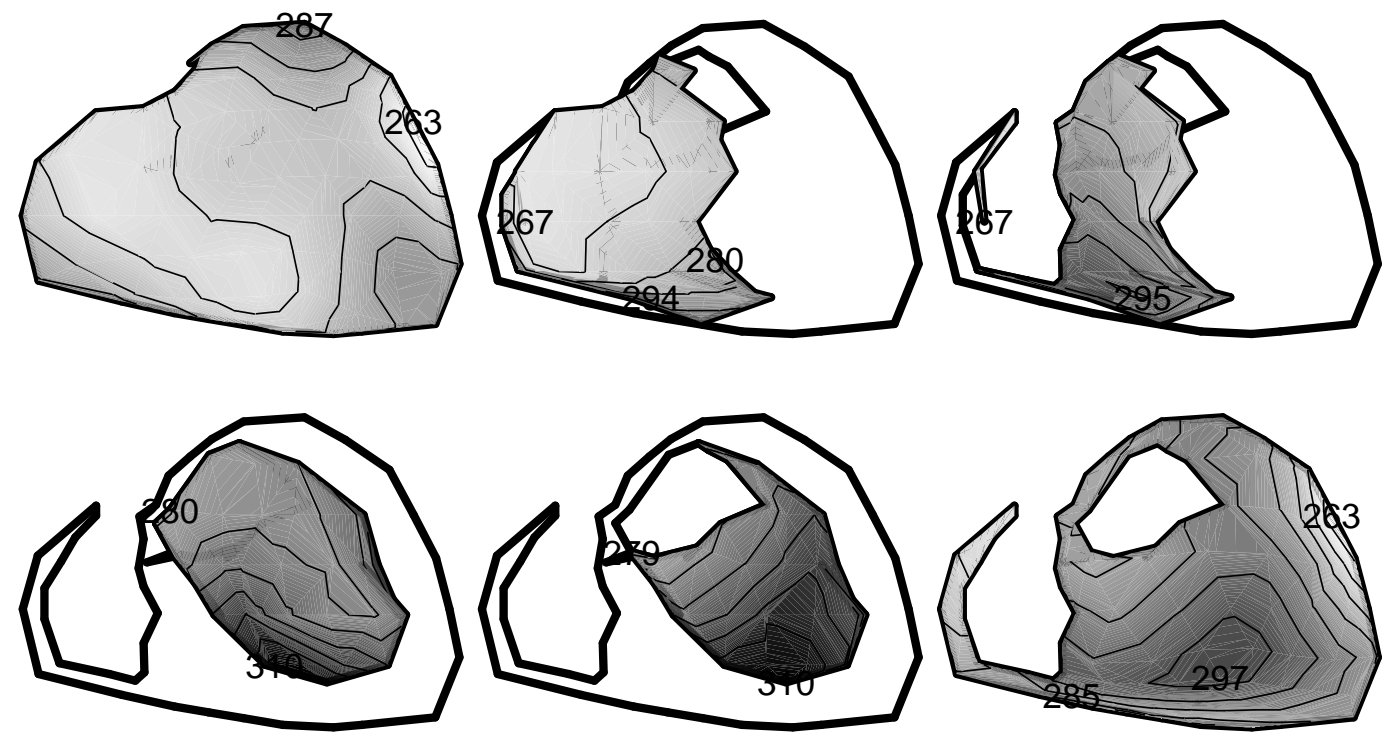

Fig. 4. Isochrone map of the inversely computed timing of repolarization, $\boldsymbol{\rho}$. Remaining legend as in Fig. 3

\section{A. Dispersion}

The dispersion of the timing of depolarization and of repolarization have received considerable attention in the literature, in particular the concept of QT dispersion. In several of these studies attempts have been made to use a measure to quantitate dispersion derived from potentials measured on the body surface. This problem is now discussed against the background of the equivalent surface source model discussed in this paper.

As shown in this paper the determination of the timing of depolarization $\boldsymbol{\delta}$ and repolarization $\boldsymbol{\rho}$ on $S_{\mathrm{h}}$ can be derived from observed body surface potentials by treating the topic as an inverse problem. The isochrone maps shown in Fig. 3 and Fig. 4 demonstrate that these solutions have a similar complexity. To use a single measure, like dispersion, for quantifying these phe- nomena is clearly a crude approach. Even so, solving the inverse problem is no simple job and if some crude measure would prove to be diagnostically helpful just the same, so much the better.

Repolarization is a continuous process that, in the normal case, may take about $300 \mathrm{~ms}$ (see Fig. 1). The peak of the curve shown in Fig. 2 identifies the mean $-\rho$ of the indicators of the timing of local repolarization: $\rho_{n}$. The vector of the differences between the $\rho_{n}$ values and $-\rho, \Delta \boldsymbol{\rho}$, is the true indicator of dispersion. As a crude measure for the inhomogeneity of repolarization (dispersion) one might take either the standard deviation (SD) or the range of the elements $\rho_{n}$, as listed in Table 1. This table shows that both measures are much smaller than the duration of the repolarization process itself. 
For the timing of the depolarization process one may similarly take SD or range as a measure of dispersion. Here the duration of the up-slope of $\varphi_{m}$ (for the discretized surface elements: 4 $\mathrm{ms}$ ) is much smaller than either the SD or the range.

The expression of dispersion of the timing at $S_{\mathrm{h}}$ in the potentials on the body surface is molded by the volume conductor effects, specified by $\mathbf{A}$. The consequence of this is that no single on-set or off-set in any signal observed on the body surface can be interpreted uniquely in terms of an event on $S_{\mathrm{h}}$. The on-sets of the potentials measured on the body surface mainly identify the earliest moment of depolarization, the termination of observed $\mathrm{T}$ waves are drawn out versions of the activities of the sections of $S_{\mathrm{h}}$ repolarizing last.

We will now use the theory unfolded here to make the statement on repolarization more specific. Starting point is Eqn. 16 which shows that, in a first order approximation, the $\mathrm{T}$ waves in all leads have the same shape, $S^{\prime}\left(t ;{ }^{-} \rho\right)$, and have amplitudes that are linear combinations of the individual $\Delta \rho_{n}$ values at the nodes on $S_{\mathrm{h}}$. One might expect that for small values of the dispersion $\Delta \boldsymbol{\rho}$, the first order approximation might be reasonable, but that for larger values the idea would fail. In order to examine this we now introduce $\boldsymbol{\rho}(a)$ as $\boldsymbol{\rho}(a)=-\boldsymbol{\rho}+a(\boldsymbol{\rho}-\boldsymbol{\rho})=-\boldsymbol{\rho}+a \Delta \boldsymbol{\rho}$ with $\boldsymbol{\rho}$ and $-\boldsymbol{\rho}$ the same as in the solution shown in Fig. 4 and $a$ a factor used for scaling the magnitude of the dispersion. For several values of $a$, T wave matrixes $\boldsymbol{\Phi}(a)$ were simulated based on the full, non-linear expression Eqn. 10:

$\boldsymbol{\Phi}(a)=\mathbf{A} \mathbf{S}\left\{{ }^{-} \boldsymbol{\rho}+a \Delta \boldsymbol{\rho}\right\}$

In addition, for the same values of $a$, T waves $\boldsymbol{\Psi}(a)$ were computed:

$\boldsymbol{\Psi}(a)=\mathbf{A D}\{a \Delta \boldsymbol{\rho}\} \mathbf{S}^{\prime}\left\{{ }^{-} \boldsymbol{\rho}\right\}=a \mathbf{A D}\{\Delta \boldsymbol{\rho}\} \mathbf{S}\left\{{ }^{-} \boldsymbol{\rho}\right\}$,

based on the linear approximation, Eqn. 15. Matrixes $\mathbf{S}$ and $\mathbf{S}^{\prime}$ were the ones found in the last iteration step of the algorithm used for finding $\boldsymbol{\rho}$. The analysis revealed that the amplitudes of the signals $\boldsymbol{\Phi}(a)$ were almost linearly proportional with the scaling factor $a$ for values of $a$ up to $a=2$. For $\boldsymbol{\Psi}(a)$ a linear dependency on $a$ for all values of $a$ holds true for all values of $a$ by virtue of Eqn. 18. Wave form differences between $\boldsymbol{\Phi}(a)$ and $\boldsymbol{\Psi}(a)$ increased for increasing values of $a\left(r d(1)=\|\boldsymbol{\Psi}(1)-\boldsymbol{\Phi}(1)\|_{F} /\|\boldsymbol{\Phi}(1)\|_{F}=0.150\right.$, and $\left.r d(2)=\|\boldsymbol{\Psi}(2)-\boldsymbol{\Phi}(2)\|_{\mathrm{F}} /\|\boldsymbol{\Phi}(2)\|_{\mathrm{F}}=0.579\right)$.

\section{B. QRST and other time integrals; Wilson's gradient}

The formalism of Eqn. 9 provides a direct view on the time integrals of ECG signals taken over either the QRST , the QRSor the ST interval. Expressed in terms of linear algebra integration over time can be effected (approximated) by means of a multiplication by a unit vector e having as its dimension the number of time samples involved.

$I(\mathrm{QRST})$, the integral over the QRST interval, was introduced by Wilson [23]; its value is known as "Wilson's ventricular gradient". In terms of Eqn. 9 we have $I(\mathrm{QRST})=\mathbf{V} \mathbf{e}=$ A S $\{\boldsymbol{\delta}, \boldsymbol{\rho}\}$ e. By inspecting Fig. 1 it is found that the time integral of the source strength for any node $n$ is approximately equal to $\rho_{n}-\delta_{n}$, which means that $\mathbf{S}\{\boldsymbol{\delta}, \boldsymbol{\rho}\} \mathbf{e}=\boldsymbol{\rho}-\boldsymbol{\delta}$ and so we find $I(\mathrm{QRST})=\mathbf{A}(\boldsymbol{\rho}-\boldsymbol{\delta})=\mathbf{A} \boldsymbol{\alpha}$. As a consequence of the special nature of matrix A (Eqn. 8) the mean action potential duration, the mean of the elements of $\boldsymbol{\alpha}$, has no effect on $I(\mathrm{QRST})$. In terms of the notation of Sect. V-A this means that

$I(\mathrm{QRST})=\mathbf{A}\left({ }^{-} \boldsymbol{\alpha}+\Delta \boldsymbol{\alpha}\right)=\mathbf{A} \Delta \boldsymbol{\alpha}$.

Since $\mathbf{A}(\boldsymbol{\rho}-\boldsymbol{\delta})=\mathbf{A} \boldsymbol{\rho}-\mathbf{A} \boldsymbol{\delta}$ we find that similarly, $I(\mathrm{QRS})=$ $\mathbf{V}_{\mathrm{d}} \mathbf{e}=-\mathbf{A} \Delta \boldsymbol{\delta}$, and $I(\mathrm{ST})=\mathbf{V}_{\mathrm{r}} \mathbf{e}=\mathbf{A} \Delta \boldsymbol{\rho}$, again with the constant mean vectors "eaten up" by $\mathbf{A}$.

\section{Local ischemia}

Another application of the formalism is that of simulating the effect of local ischemia. This can be effected by assigning a zero value to the source strengths of the nodes of the ischemic part of $S_{\mathrm{h}}$. As an illustration, the results are shown here of the situation where three nodes representing an epicardial region $S_{\text {epi }}$, an anterior epicardial subregion of $S_{\mathrm{h}}$ above the septum projecting on the thorax at about $3 \mathrm{~cm}$ above the level of lead V3, were ,assigned zero strength while leaving all other nodes unaffected. The consequence of this is demonstrated in Fig. 6. Shown are the measured and simulated signals of six of the standard 12lead ECGs. The solid lines are the measured signals (without ischemia), the dashed lines are the signals that would be generated if the ischemic region were active by itself. The dash-dot lines depict the signals generated by the remaining part of $S_{\mathrm{h}}$. These signals arise if the source strength at $S_{\text {epi }}$ would be wiped out completely during an ischemic period, and can be found simply by subtracting the dashed curves from the solid ones. Gradual development of such events can be modeled by simply scaling the source strength of the affected region by a factor $f$, with $0 \leq f \leq 1$.

The underlying assumption is that the timing at the other part of $S_{\mathrm{h}}$ remains the same. For more widespread ischemia the essence of the signal generation by the model is still valid, but the timing will be affected and, hence, the vectors of timing, $\boldsymbol{\delta}$ and $\boldsymbol{\rho}$, must be adapted accordingly.

\section{DISCUSSION}

This paper presents a matrix formalism, Eqn. 7, of equivalent surface source models, which is valid for the simulation of the potentials on the thorax throughout the entire cardiac cycle. The volume conduction effects are expressed by means of the transfer matrix A. The UDL-based source model studied in this paper is just an example of such equivalent source models, the pericardial (epicardial) potential based model being another one (see, e.g., [9], [19]).

If the source description used is the equivalent double layer the mean of the source strength over the closed surface $S_{\mathrm{h}}$ does not at any time generate any potential differences in the region exterior to the heart. This was expressed by means of Eqn. 8.

With assumed uniform strength, the UDL approach, the forward problem of computing body surface potentials can be presented by Eqn. 9, through which body surface potentials can be derived from the timing of depolarization and repolarization at the heart surface. In this paper, mainly aimed at describing the 
1.6

$\mathrm{aVR}$

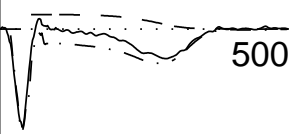

$-1.6$

1.6

V1

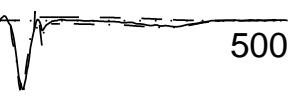

$-1.6$

1.6

V3

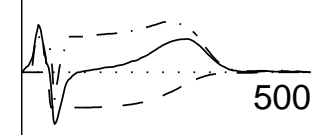

$-1.6$

$-1.6$

Fig. 6. Measured and simulated signals of six of the standard 12-lead ECGs; solid lines: measured signals; dashed lines: simulated signals generated by just $S_{\text {epi }}$; dash-dot lines: signals generated by the remaining part of $S_{\mathrm{h}}$. Voltage scale: $1.6 \mathrm{mV}$; time scale: $500 \mathrm{~ms}$.

normal situation, the shape of the $\mathrm{T}$ wave was specified by a single curve for describing a "mean" transmembrane potential during repolarization (Fig. 1), which was estimated as an integral over time of the "mean" $\mathrm{T}$ wave forms as observed on the thorax. This approach is valid as long as the inhomogeneity in repolarization times (dispersion) is not too great. If local differences in the actual waveforms exist, local variants of the source strength at each individual node need to be specified.

Within the range of its validity, the matrix formalism Eqn. 9 enables the inverse computation of the timing of depolarization and repolarization at $S_{\mathrm{h}}$ from measured body surface potentials and a known transfer function $\mathbf{A}$ describing the volume conduction effects (Eqn. 14). The inversely computed isochrones of depolarization correspond well with invasively obtained data. The computed timing of repolarization is of a nature that agrees with the fragmented data reported in the literature obtained through invasive measurements. The analysis of the first order expansion of source strength (Eqns. 15 and 16) leads to an estimate of the down-slope of the transmembrane potential, derived from $T$ waves observed on the body surface. The UDL model leads to Eqn. 19, which shows that the QRST integrals of surface leads (Wilson's gradient) are weighted combinations of the dispersion of the APD values at the nodes of $S_{h}$ rather than of the APD values themselves. This result extends previous theoretical considerations on this topic (see [24], [25], [26]). Note that this result is largely independent of the actual shape of the recovery phase of $\varphi_{m}$.

The following comments can be expected to be valid even if the solution vector, $\boldsymbol{\rho}$ as depicted in Fig. 4, may prove to be sub-optimal.

It was demonstrated that the shape of the $\mathrm{T}$ wave is relatively insensitive to the magnitude of the dispersion of the timing of repolarization, but this magnitude is shown to be proportional to the peak values of the $\mathrm{T}$ waves on the body surface. The constant of proportionality itself depends on the volume conductor properties, A.

The model provides a straightforward manner for simulating the effect of local ischemia, see Fig.6. The results suggest that the elevations or depressions directly following QRS are the clearest markers for identifying the site of the ischemia. The subsequent development of the $\mathrm{T}$ wave largely obscures it. The timing of the occurrence of these shifts corresponds to the timing at which the region $S_{\text {epi }}$ would have depolarized prior to ischemia.

If the source strengths of the "ischemic nodes" are assigned a constant positive value, then the simulated ECGs will show the DC-shifts following ischemia[27]. These shifts commonly remain unobserved when measuring ECG signals due to the ACcoupling to the ECG amplifiers followed by assigning a zero value to the baseline.

\section{APPENDIX}

Proof of the equation preceeding Eqn. 14.

We first rewrite Eqn. 13, in terms of the preceeding resiual $\mathbf{R}$, as

$\mathbf{R}=\mathbf{A} \mathbf{D}\{\Delta \boldsymbol{\rho}\} \mathbf{G}$,

while dropping the sub-indices, and denoting the gradients of the source term, $\mathbf{S}^{\prime}$, as $\mathbf{G}$. The perturbation $\Delta \boldsymbol{\rho}$ is found by minimizing the sum of the squares of all $L \times T$ differences $r_{l, t}-\sum_{n} a_{l, n} \Delta \rho_{n} g_{n, t}$. Equating to zero the partial derivatives of this sum of squares with respect to $\Delta \rho_{k} ; k=1, N$ amounts to solving:

$\frac{\partial E^{2}}{\partial \Delta \rho_{k}}=\frac{\partial}{\partial \Delta \rho_{k}} \sum_{l} \sum_{t}\left(r_{l, t}-\sum_{n} a_{l, n} \Delta \rho_{n} g_{n, t}\right)^{2}=0$

Since $\frac{\partial \Delta \rho_{n}}{\partial \Delta \rho_{k}}=1$ for $n=k$ and zero otherwise, the previous equation works out as

$\frac{\partial E^{2}}{\partial \Delta \rho_{k}}=2 \sum_{l} \sum_{t}\left(r_{l, t}-\sum_{n} a_{l, n} \Delta \rho_{n} g_{n, t}\right) a_{l, k} g_{k, l}=0$.

By reordering and applying subsequent changes in summation order we find

$$
\begin{aligned}
\sum_{l} \sum_{t} \sum_{n} a_{l, n} \Delta \rho_{n} g_{n, t} a_{l, k} g_{k, t} & =\sum_{l} \sum_{t} r_{l, t} a_{l, k} g_{k, t} \\
\sum_{n} \Delta \rho_{n} \sum_{l} \sum_{t} a_{l, n} g_{n, t} a_{l, k} g_{k, t} & =\sum_{l} a_{l, k} \sum_{t} r_{l, t} g_{k, t} \\
\sum_{n} \Delta \rho_{n} \sum_{l} a_{l, n} a_{l, k} \sum_{t} g_{n, t} g_{k, t} & =\sum_{l} a_{l, k}\left(\mathbf{R G}^{T}\right)_{l, k}
\end{aligned}
$$


which can be denoted as

$$
\left(\mathbf{A}^{T} \mathbf{A} \cdot * \mathbf{G G}^{T}\right) \Delta \boldsymbol{\rho}=\left(\mathbf{G R}^{T} \cdot * \mathbf{A}^{T}\right) \mathbf{e}
$$

q.e.d. .

\section{REFERENCES}

[1] B. C. Surawicz, "ST-T Abnormalities," in Comprehensive Electrocardiology (P. W. Macfarlane and T. T. V. Lawrie, eds.), ch. 15, pp. 511-563, Oxford: Pergamon Press, 1989.

[2] R. C. Barr, "Genesis of the electrocardiogram," in Comprehensive Electrocardiology (P. W. Macfarlane and T. T. V. Lawrie, eds.), ch. 5, pp. 129-151, Oxford: Pergamon Press, 1989.

[3] K. Harumi, M. J. Burgess, and J. A. Abildskov, "A Theoretic Model of the T Wave," Circulation, vol. XXIV, pp. 657-668, 1966.

[4] D. B. Geselowitz, "On the Theory of the Electrocardiogram," Proceedings IEEE, vol. 77/6, pp. 857-876, 1989.

[5] D. B. Geselowitz, "Description of cardiac sources in anisotropic cardiac muscle. Application of bidomain model," Journal of Electrocardiology, vol. 25 Sup., pp. 65-67, 1992.

[6] G. J. M. Huiskamp and A. van Oosterom, "The depolarization sequence of the human heart surface computed from measured body surface potentials," IEEE Trans. Biomed. Eng., vol. BME-35, pp. 1047-1058, 1988.

[7] A. van Oosterom and G. J. M. Huiskamp, "The effect of torso inhomogeneities on body surface potentials quantified by using tailored geometry," J Electrocardiol, vol. 22, pp. 53-72, 1989.

[8] A. van Oosterom, G. Windau, and G. J. M. Huiskamp, "Simulation on a PC of the QRS-T wave forms," in Electrocardiology '93 (P. W. Macfarlane and P. Rautaharju, eds.), (Singapore), pp. 97-100, World Scientific, 1994.

[9] R. M. Gulrajani, Bioelectricity and Biomagnetism. New York: John Wiley \& Sons, 1998

[10] P. Colli-Franzone, L. Guerri, and S. Rovida, "Wavefront propagation in an activation model of the anisotropic cardiac tissue: Asymptotic analysis and numerical solutions," J. Math. Biol., vol. 28, pp. 121-176, 1990.

[11] A. van Oosterom and R. T. van Dam, "Potential distribution in the left ventricular wall during depolarization," Adv. Cardiology, vol. 16, pp. 2731, 1976.

[12] A. van Oosterom, "Cell Models - Macroscopic Source Descriptions," in Comprehensive Electrocardiology (P. W. Macfarlane and T. T. V. Lawrie, eds.), vol. I, ch. 6, pp. 155-179, Oxford: Pergamon Press, 1989.

[13] R. Plonsey and A. van Oosterom, "Implications of macroscopic source strength on cardiac cellular activation models," J. Electrocardiology., vol. 24/2, pp. 99-112, 1991.

[14] J. J. M. Cuppen and A. van Oosterom, "Model studies with the inversely calculated isochrones of ventricular depolarization," IEEE Trans. Biomed. Eng., vol. BME-31, pp. 652-659, 1984.

[15] F. N. Wilson, A. G. Macleod, and P. S. Barker, "The Distribution of Action Currents produced by the Heart Muscle and Other Excitable Tissues immersed in Conducting Media," J. Gen. Physiol., vol. 16, pp. 423-456, 1933.

[16] H. Simms and D. Geselowitz, "Computation of Heart Surface Potentials Using the Surface Source Model," J. Cardiovasc. Electrophysiol., vol. 6, pp. 522-531, 1995.

[17] G. J. M. Huiskamp, "Simulation of Depolarization and Repolarization in a Membrane Equations based Model of the Anisotropic Ventricle," IEEE$B M E$, vol. $45 / 7$, pp. $847-855,1998$.

[18] A. Heringa, G. J. H. Uijen, and R. T. van Dam, "A 64-channel system for body surface potential mapping," in Electrocardiology 1981 (Z. Antalózcy and I. Préda, eds.), (Budapest, Hungary), pp. 297-301, Academia Kiado, 1982.

[19] A. van Oosterom, "The Spatial Covariance used in Computing the Pericardial Potential Distribution," in Computational Inverse Problem of Electrocardiography (P. R. Johnston, ed.), vol. Advances in Computational Biomedicine 3, pp. 1-50, Southhampton; U.K: Computational Mechanics Publications, 2001. Chapter 1.

[20] M. Franz, K. Bargheer, W. Rafflenbeul, A. Haverich, and P. Lichtlen, "Monophasic action potential mapping in a human subject with normal electrograms: direct evidence for the genesis of the T wave," Circulation, vol. $75 / 2$, pp. 379-386, 1987.

[21] D. W. Marquardt, "An algorithm for least-squares estimation of non-linear parameters," J. Soc. Indust. Appl. Math., vol. 2, pp. 431-441, 1963.

[22] D. Durrer, R. T. van Dam, G. E. Freud, M. J. Janse, F. L. Meijler, and R. C. Arzbaecher, "Total excitation of the isolated human heart," Circulation, vol. 41, pp. 899-912, 1970 .
[23] F. N. Wilson, A. G. Macleod, P. S. Barker, and F. D. Johnston, "The Determination and Significance of the Areas of the Ventricular Deflections of the Electrocardiogram," Am. Heart J., vol. 10, pp. 46-61, 1934.

[24] H. C. Burger, "A Theoretical Elucidation of the Notion: Ventricular Gradient," Am. Heart J., vol. 53/2, pp. 240-246, 1957.

[25] R. Plonsey, "A Contempory View of the Ventricular Gradient of Wilson," J. Electrocardiology., vol. 12/4, pp. 337-341, 1979.

[26] D. B. Geselowitz, "The Ventricular Gradient Revisited: Relation to the Area Under the Action Potentials," IEEE Trans. Biomed. Eng., vol. BME30/1, pp. 76-77, 1983.

[27] A. G. Kléber, J. Janse, F. J. L. van Capelle, and D. Durrer, "Mechanism and time course of S-T and T-Q segment changes during acute regional myocardial ischemia in the pig heart determinated by extracellular and intracellular recordings," Circulation Res., vol. 42, pp. 603-613, 1978. 